\title{
Spherical collapse of supermassive stars: Neutrino emission and gamma-ray bursts
}

\author{
F. Linke ${ }^{1}$, J. A. Font ${ }^{1}$, H.-T. Janka ${ }^{1}$, E. Müller ${ }^{1}$, and P. Papadopoulos ${ }^{2}$ \\ 1 Max-Planck-Institut für Astrophysik, Karl-Schwarzschild-Str. 1, 85741 Garching, Germany \\ 2 School of Computer Science and Mathematics, University of Portsmouth, P01 2EG, Portsmouth, UK
}

Received 9 March 2001 / Accepted 6 July 2001

\begin{abstract}
We present the results of numerical simulations of the spherically symmetric gravitational collapse of supermassive stars (SMS). The collapse is studied using a general relativistic hydrodynamics code. The coupled system of Einstein and fluid equations is solved employing coordinates adapted to a foliation of the spacetime by means of outgoing null hypersurfaces. The code contains an equation of state which includes effects due to radiation, electrons and baryons, and detailed microphysics to account for electron-positron pairs. In addition energy losses by thermal neutrino emission are included. We are able to follow the collapse of SMS from the onset of instability up to the point of black hole formation. Several SMS with masses in the range $5 \times 10^{5} M_{\odot}-10^{9} M_{\odot}$ are simulated. In all models an apparent horizon forms initially, enclosing the innermost $25 \%$ of the stellar mass. From the computed neutrino luminosities, estimates of the energy deposition by $\nu \bar{\nu}$-annihilation are obtained. Only a small fraction of this energy is deposited near the surface of the star, where, as proposed recently by Fuller \& Shi (1998), it could cause the ultrarelativistic flow believed to be responsible for $\gamma$-ray bursts. Our simulations show that for collapsing SMS with masses larger than $5 \times 10^{5} M_{\odot}$ the energy deposition is at least two orders of magnitude too small to explain the energetics of observed long-duration bursts at cosmological redshifts. In addition, in the absence of rotational effects the energy is deposited in a region containing most of the stellar mass. Therefore relativistic ejection of matter is impossible.
\end{abstract}

Key words. hydrodynamics - methods: numerical - relativity - gamma ray bursts - elementary particles - neutrinos

\section{Introduction}

Recent observations with the Space Telescope Imaging Spectrograph on board the HST have added stronger support to the increasing belief that supermassive black holes $(\mathrm{SMBH})$ are not unusual in nature: the central objects in more than 45 galaxies have been examined and in 34 of them the presence of a SMBH has been confirmed (Kormendy 2000; see also Rees 1998 for an overview of SMBH). The inferred masses of these objects imply that SMBH of $10^{6}-10^{9} M_{\odot}$ are present in the center of probably most, if not all, galaxies. Our own Galaxy with a central mass between $2.6 \times 10^{6} M_{\odot}$ and $3.3 \times 10^{6} M_{\odot}$ (Genzel et al. 2000) lies at the lower limit of this mass range. In the particular cases of NGC 4258 and our Galaxy, the radius inferred for the location of the "dark mass" is sufficiently small to exclude any black hole alternative (Maoz 1998). The question of how SMBH form, is, however, still not settled and the nature of their progenitors is still rather uncertain (see, e.g., Rees 1984). Two different scenarios

Send offprint requests to: J. A. Font,

e-mail: font@mpa-garching.mpg.de have essentially been proposed, relying upon either stellar dynamics in a dense star cluster or on the hydrodynamics of a single supermassive star (SMS). Both cases can develop a dynamical instability (Chandrasekhar 1964; Fowler 1964; Shapiro \& Teukolsky 1985) and can therefore undergo catastrophic gravitational collapse which would lead to the formation of a SMBH.

Despite missing theoretical and observational evidence for the existence of SMS, it is still of theoretical interest to study their properties, due to their potential astrophysical relevance as progenitors of SMBH. In particular, it is worth analyzing the dynamics involved in the gravitational collapse of such stars. The low frequency gravitational waves emitted during such (in cases of rotating stars) events could be detected by the proposed Laser Interferometer Space Antenna (LISA). Strong bursts of gravitational radiation would only occur for homologous collapse down to small radii, but not in cases of the formation of an initially small black hole which gradually grows through subsequent accretion (Thorne \& Braginsky 1976).

A number of collapse simulations of SMS have been attempted through the years. The first ones were done by 
Appenzeller \& Fricke (1972a,b) who studied the collapse of spherical stars of masses $7.5 \times 10^{5} M_{\odot}$ and $5.2 \times 10^{5} M_{\odot}$, using a code with only a subset of the general relativistic equations implemented. Since core temperatures higher than $5 \times 10^{8} \mathrm{~K}$ were encountered, nuclear burning was expected to release high amounts of energy. They also found that for stars with masses greater than $10^{6} M_{\odot}$ thermonuclear reactions have no major effect on the evolution. In a subsequent paper Fricke (1973) studied the fate of SMS depending on the initial metallicity and mass. Later, Shapiro \& Teukolsky (1979) simulated the evolution of a $10^{6} M_{\odot}$ star with a spherically-symmetric relativistic code which did not include any microphysics effects. They were able to follow the whole evolution of the collapsing star until the formation of a black hole. Fuller et al. (1986) revisited the work of Appenzeller \& Fricke performing simulations in the range of $10^{5}-10^{6} M_{\odot}$ with a code which included post-Newtonian corrections and detailed microphysics. They found that SMS with zero initial metallicity do not explode, but the critical metallicity for an explosion was considerably reduced compared to the results of Appenzeller \& Fricke. In a series of recent papers, Baumgarte \& Shapiro (1999a, b) investigated the influence of rotation on the evolution and luminosity of a SMS, finding that the luminosity is reduced by rotation and, hence, the lifetime of the star is enhanced. Furthermore, they showed that the ratios $R / M, T /|W|$ and $J / M^{2}$, where $R$ is the radius, $M$ the mass, $T$ the rotational energy, $J$ the angular momentum and $W$ the gravitational potential energy of the star, are universal numbers at the onset of instability. Therefore they concluded that the collapse of SMS should produce universal gravitational waveforms.

SMS have recently re-gained theoretical interest since Fuller \& Shi (1998) proposed a scenario to explain the origin of cosmological $\gamma$-ray bursts (GRBs) from the collapse of SMS. The large amounts of emitted thermal neutrinos and antineutrinos during the catastrophic collapse of such stars (or, alternatively, of stellar clusters) could deposit a significant fraction of their energy by annihilation to electron-positron pairs near the surface of the star. They argued that this annihilation-induced heating could lead to relativistic expansion and associated $\gamma$-ray emission by cyclotron radiation and/or by the inverse Compton process, producing the required energies for a GRB in the range $10^{51}-10^{54}$ ergs within several seconds (assuming isotropic emission).

In order to give reliable, quantitative estimates of the neutrino emission from the collapse of SMS we have performed a number of numerical simulations of spherically symmetric collapsing SMS, using a relativistic hydrodynamics code (Papadopoulos \& Font 2000a). The code is able to follow the collapse from the onset of instability up to the point of black hole formation. This was an important consideration since, due to the large temperature dependence of the neutrino emission, most of the neutrinos are emitted close to the onset of black hole formation, when the temperatures in the stellar core are highest.
This paper is organized as follows: in Sect. 2 we present the problem setup, describing the equation of state we use, the equations of general relativistic hydrodynamics and the initial data for SMS. We also discuss some computational issues relevant to our simulations. These simulations are presented and analyzed in Sect. 3, together with some tests to show the accuracy of the numerical code. Moreover, this section contains our results concerning the neutrino emission and the energy deposition by neutrinoantineutrino annihilation during SMS collapse. In Sect. 5 we discuss the implications of our results for GRBs. The paper ends with a summary in Sect. 6 .

\section{Problem setup}

\subsection{Properties of SMS and microphysics}

SMS are supported against gravitational collapse by radiation pressure, the gas pressure being negligible. Such stars are very well approximated in equilibrium by polytropes, $p=K \rho^{\Gamma}, \Gamma=1+1 / n$ with polytropic index $n=3$ $(\Gamma=4 / 3)$.

SMS have a two-dimensional parameter space: the central density $\rho_{\mathrm{c}}$, which controls the radius of the star, and the mass of the star $M$, which determines the ratio $\tilde{\beta}$ between gas pressure and total pressure via Eddington's quartic equation (Kippenhahn \& Wigert 1994)

$3.02 \times 10^{-3}\left(\frac{M}{M_{\odot}}\right)^{2}=\frac{1-\tilde{\beta}}{\mu^{4} \tilde{\beta}^{4}}$,

where $\mu$ is the mean molecular weight. The polytropic constant $K$ is related to $\tilde{\beta}$ by

$K=\left(\frac{3 \mathcal{R}^{4}}{a \mu^{4}}\right)^{\frac{1}{3}}\left(\frac{1-\tilde{\beta}}{\tilde{\beta}^{4}}\right)^{\frac{1}{3}}$

where $\mathcal{R}$ is the universal gas constant and $a$ is the radiation constant.

The adiabatic index is slightly larger than $4 / 3$ due to the contribution from baryons. The onset of instability occurs when the adiabatic index drops below a critical value

$\Gamma_{\text {crit }}^{\prime}=\frac{4}{3}+1.12 \frac{2 G M}{R c^{2}}$

where $G$ is the gravitational constant and $c$ is the speed of light. This corresponds to a critical central density (Chandrasekhar 1964)

$\rho_{\text {crit }}=1.994 \times 10^{18}\left(\frac{0.5}{\mu}\right)^{3}\left(\frac{M_{\odot}}{M}\right)^{\frac{7}{2}} \mathrm{~g} \mathrm{~cm}^{-3}$

Stars with a central density $\rho_{\mathrm{c}}$ larger than $\rho_{\text {crit }}$ will become dynamically unstable.

In our code we have implemented a tabulated equation of state (EOS) $p=p(\rho, \epsilon)$ which includes radiation, electrons and effects associated with the creation of electronpositron pairs. Contributions due to the presence of an 


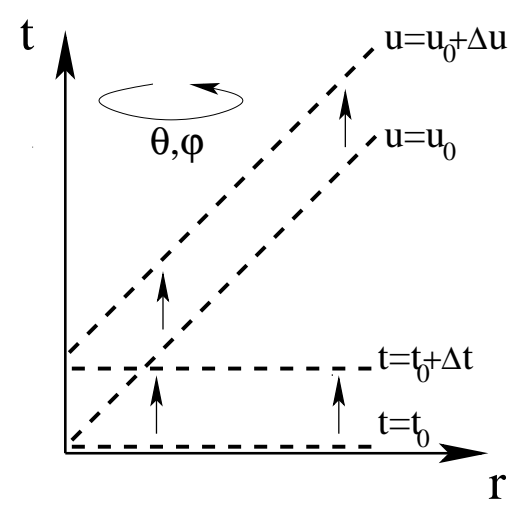

Fig. 1. In the $3+1$ formulation initial data are constructed on spacelike hypersurfaces, $t \equiv$ const. In the characteristic formulation employed in this work, initial data are constructed on null hypersurfaces (lightcones), $u=$ const.

additional Boltzmann gas $\left(\mathrm{H}^{+}\right)$are taken into account in an appropriate way. Given $\epsilon$, the specific internal energy, and $\rho$, it is possible to determine the temperature $T$ by a Newton-Raphson iteration and then compute the pressure from $\rho$ and $T$.

Furthermore, the EOS allows us to compute neutrino energy loss rates due to pair annihilation, $e^{-}+e^{+} \rightarrow \nu+\bar{\nu}$, photo-neutrino emission, $\gamma+e^{ \pm} \rightarrow e^{ \pm}+\nu+\bar{\nu}$, and plasmon decay $\gamma \rightarrow \nu+\bar{\nu}$. The fitting formulas and tables for the neutrino energy loss rates of the first two processes have been taken from Itoh et al. (1996). The corresponding expressions for plasmon decay were obtained from Haft et al. (1994). These processes become relevant in the very last epochs of the collapse.

\subsection{General relativistic hydrodynamics}

The formulation and implementation of the Einstein and hydrodynamic equations follows the work of Papadopoulos and Font (2000a,b). These equations are formulated adopting an outgoing null foliation of the spacetime (Bondi et al. 1962; Sachs 1962), whose line element, in spherical symmetry reads:

$\mathrm{d} s^{2}=-\frac{e^{2 \beta} V}{r} \mathrm{~d} u^{2}-2 e^{2 \beta} \mathrm{d} u \mathrm{~d} r+r^{2}\left(\mathrm{~d} \theta^{2}+\sin ^{2} \theta \mathrm{d} \phi^{2}\right)$.

The radial coordinate $r$ is chosen to make the spheres of rotational symmetry have an area of $4 \pi r^{2}$. The retarded time $u$ labels the null cones in terms of proper time along the central geodesic. Using this time coordinate the computed profiles of scalar quantities, like the density and the pressure, are the same as if seen by an observer at infinity. Additionally, $\theta$ and $\phi$ are angular coordinates for the null rays, as shown in Fig. 1. The geometry is completely described by the two metric functions $V(u, r)$ and $\beta(u, r)$.

The hydrodynamic equations are cast into a first-order, flux-conservative system which, in spherical symmetry, reads:

$\partial_{u} U+\frac{1}{\sqrt{-g}} \partial_{r} \sqrt{-g} F=S$,
Table 1. Parameters of the initial models: Mass and central density. The criterion for the onset of dynamical instability, Eq. (4), gives the central densities.

\begin{tabular}{|c|c|}
\hline $\begin{array}{l}\text { Mass } \\
{\left[M_{\odot}\right]}\end{array}$ & $\begin{array}{c}\rho_{c} \\
{\left[\mathrm{~g} / \mathrm{cm}^{3}\right]}\end{array}$ \\
\hline $5 \times 10^{5}$ & $2.26 \times 10^{-2}$ \\
\hline $1 \times 10^{6}$ & $2.80 \times 10^{-3}$ \\
\hline $5 \times 10^{6}$ & $7.14 \times 10^{-6}$ \\
\hline $1 \times 10^{7}$ & $6.30 \times 10^{-7}$ \\
\hline $5 \times 10^{7}$ & $2.26 \times 10^{-9}$ \\
\hline $1 \times 10^{8}$ & $1.99 \times 10^{-10}$ \\
\hline $1 \times 10^{9}$ & $6.30 \times 10^{-14}$ \\
\hline
\end{tabular}

with

$$
\begin{aligned}
& U=\left(D, S^{r}, E\right), \\
& F^{r}=\left(\rho u^{r}, \rho h u^{r} u^{r}+p g^{r r}, \rho h u^{0} u^{r}+p g^{0 r}\right), \\
& S=\left(-\frac{D}{\sqrt{-g}} \partial_{u} \sqrt{-g},-\Gamma_{\mu \lambda}^{r} T^{\mu \lambda}-\frac{S^{r}}{\sqrt{-g}} \partial_{u} \sqrt{-g},\right. \\
& \left.-\Gamma_{\mu \lambda}^{u} T^{\mu \lambda}-\frac{E}{\sqrt{-g}} \partial_{u} \sqrt{-g}\right),
\end{aligned}
$$

where $T^{\mu \nu}$ is the stress-energy tensor, $\Gamma_{\mu \lambda}^{\nu}$ are the Christoffel symbols and $\sqrt{-g}$ is the volume element, which is given, for the chosen geometry, by $\sqrt{-g}=r^{2} e^{2 \beta} \sin ^{2} \theta$. The state vector - the conserved quantities - contains the relativistic densities of mass, momentum and energy, defined, in terms of the primitive variables, $\boldsymbol{w}=\left(\rho, u^{r}, \epsilon\right)$, as $D=\rho u^{0}, S^{r}=\rho h u^{0} u^{r}+p g^{0 r}$ and $E=\rho h u^{0} u^{0}+p g^{00}$, respectively. In this expressions $h$ is the specific enthalpy, $h=1+\epsilon+p / \rho$, and $u^{0}$ and $u^{r}$ are the time and radial components of the 4-velocity, respectively.

The Einstein equations reduce to two radial hypersurface equations for $\beta$ and $V$ :

$\beta_{, r}=2 \pi r e^{4 \beta} E$,

$V_{, r}=e^{2 \beta}-8 \pi r^{2} e^{4 \beta} S^{r}-4 \pi r e^{4 \beta} V E$.

The integration of the metric is thus completely separated from the recovery of the primitive quantities, which simplifies the recovery. Instead of a three-dimensional root finding procedure, coupled to the two ODEs for the metric, only a one dimensional problem must be solved.

\subsection{Initial data}

All SMS models in our sample are chosen such that the initial central density is a bit larger than the critical central density given by Eq. (4), for the particular mass of the star. They are listed in Table 1 . The initial models for the simulations are therefore described by one parameter only, the mass of the star.

The equations describing the hydrostatic equilibrium are theTolman-Oppenheimer-Volkoff (TOV) equations. 
Using outgoing Bondi-Sachs coordinates they read (Papadopoulos \& Font 2000a):

$$
\begin{aligned}
& p_{, r}=\left(\frac{1}{2 r}-\frac{1}{2 Y}\left(1+8 \pi r^{2} p\right)\right) \rho h, \\
& Y_{, r}=1+8 \pi r^{2}(p-\rho h),
\end{aligned}
$$

where $Y=V e^{-2 \beta}$. These coupled differential equations are integrated by a fourth-order Runge-Kutta scheme. The relation between pressure and density is prescribed by the polytropic structure relation. The integration of Eq. (10) yields $\beta$. The boundary conditions at $r=0$ are $p=p_{\mathrm{c}}=$ $K \rho_{\mathrm{c}}^{4 / 3}, Y=0$ and $\beta=0$. The polytropic constant can be computed using Eq. (2) and the corresponding $\tilde{\beta}$ is given by Eq. (1).

It may be criticized that initial data for an unstable star be prescribed on a lightcone rather than on a spacelike hypersurface. For stable stars the density profile is independent of the spacetime slicing but in dynamic situations this does not hold anymore. Other authors (Baumgarte et al. 1995) circumvent this problem. They generate initial data on null hypersurfaces by using a spacelike $3+1$ code. They trace outgoing lightrays, and store the hydrodynamic quantities encountered along its path. However, as the stellar structure does not change much during the first light-crossing time, a direct prescription on null hypersurfaces seems to be sufficient and justified.

\subsection{Computational issues}

The numerical code uses a conservative Godunov-type scheme with an approximate Riemann solver to integrate the (hyperbolic) hydrodynamic equations. The hypersurface equations (Eqs. (10) and (11)) for the metric components are solved using a two-step Runge-Kutta scheme. Specific details about the numerical algorithms can be found in Papadopoulos \& Font (2000a). The canonical Eulerian grid used for our collapse simulations of SMS consists of 500 fixed zones distributed in a non-equidistant pattern, geometrically growing away from the origin, such that the final Schwarzschild radius of the star is resolved with roughly 50 zones.

It is worth discussing in some detail the computational difficulties arising from the use of outgoing lightlike spacetime foliations when a black hole forms. We have found that in numerical simulations of collapsing SMS (see next section) a black hole forms at the center which only encompasses the innermost $\sim 25 \%$ of the total mass. The rest of the star's mass accretes on this seed black hole in a dynamical timescale. When the computation is continued beyond the moment when the black hole first appears, the density profile still looks reasonable, but the internal energy develops spikes and grows rapidly in the region $M(r) \geq 0.25 M$. The region interior to that mass does not evolve further and thus remains intact. The unphysical behavior can be postponed by severely limiting the time step near black hole formation. Similar observations were reported by Gómez et al. (1996) who simulated the evolution of a scalar field in retarded time coordinates up to the point of black hole formation.
The conditions arising at the onset of black hole formation are shown in Fig. 2 for the collapse of a pressureless (dust) star of homogeneous density. Lightrays, whose trajectories can be computed analytically, are emitted from the center of the star. They cross the surface of the collapsing star when the formation of the event horizon is about to begin and thus they suffer a severe delay.

The mass $m$ of the accreting mass shell is only important for determining the final event horizon, its trajectory being independent of this mass. When the black hole is just about to form, the current computational null hypersurface can be as close as possible to the true event horizon, this distance being highly dependent on the numerical resolution. Our numerical code advances data on outgoing null hypersurfaces. In Fig. 2, the three trajectories of the lightrays represent the last three time steps of the simulation. The region below the uppermost lightray trajectory, particularly the segments of the stellar surface and of the mass shell, drawn by solid lines, have already been computed. Without taking additional measures it is not possible to compute the dotted section of the accreting mass shell.

Other authors have reported similar problems with their implementation of methods based on a retarded time coordinate, e.g., the "observer time coordinate" method used by Baumgarte et al. (1995) in the context of the collapse of neutron stars to black holes (Baumgarte et al. 1995). In their case it helped to reduce the accuracy of their numerical method by performing a first-order accurate integration of the metric component $g_{00}$. Effectively, this first-order scheme constantly underestimates $g_{00}$, thus preventing the penetration of the horizon. In our formulation of the hydrodynamic equations it turns out that manipulating the metric while keeping the conserved quantities fixed influences the distribution of the primitive quantities in such a way that the metric component $g_{00}$ is less underestimated or even overestimated.

Our results that a black hole forms inside the collapsing SMS at about $M(r) \sim 0.25 M_{\text {SMS }}$ agree quite well with the results of other authors. Woosley et al. (1986) found a trapped surface at $M(r) \sim 0.2 M_{\mathrm{SMS}}$ for a $5 \times 10^{5} M_{\odot}$ SMS and similar results were reported by Shapiro \& Teukolsky (1979) for a $10^{6} M_{\odot}$ SMS.

Our main aim is to compute the lightcurves of neutrino emission during the collapse of a SMS. The neutrino luminosities will, like lightrays, suffer from severe redshift when black hole formation is in progress. However, as we discuss later, almost all neutrinos are emitted near the center of the star and, hence, their redshift is mostly due to the $\sim 0.25 M_{\text {SMS }}$ which gives rise to the seed black hole. The matter further outside has not yet collapsed far enough to have a significant impact on the redshift.

\section{Simulations}

We now describe our simulations, presenting first a series of tests we have performed in order to calibrate the code. 


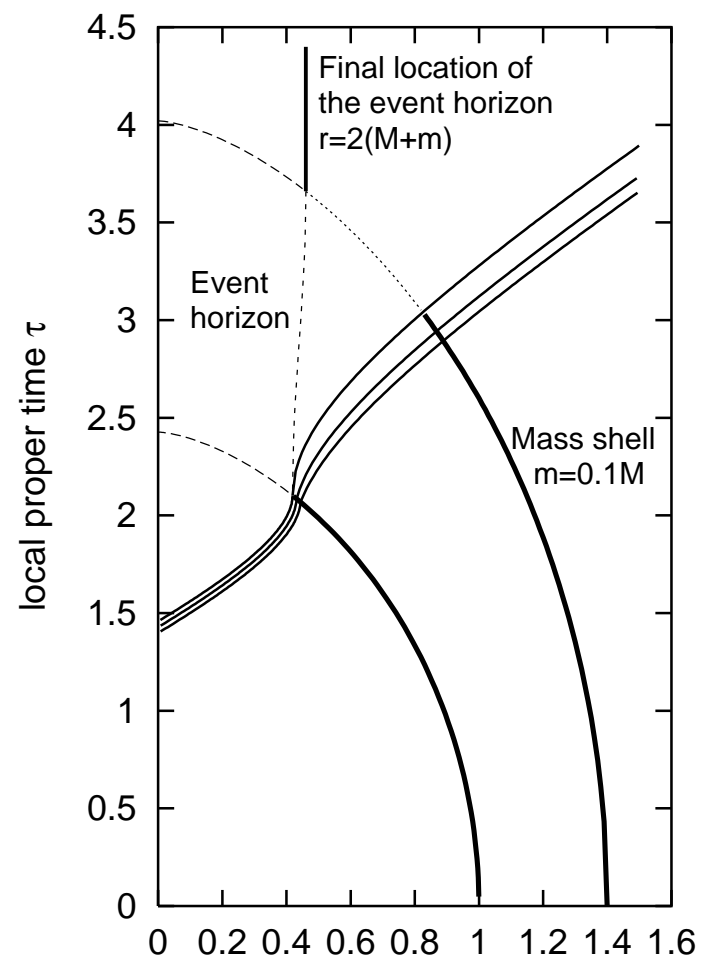

$r$
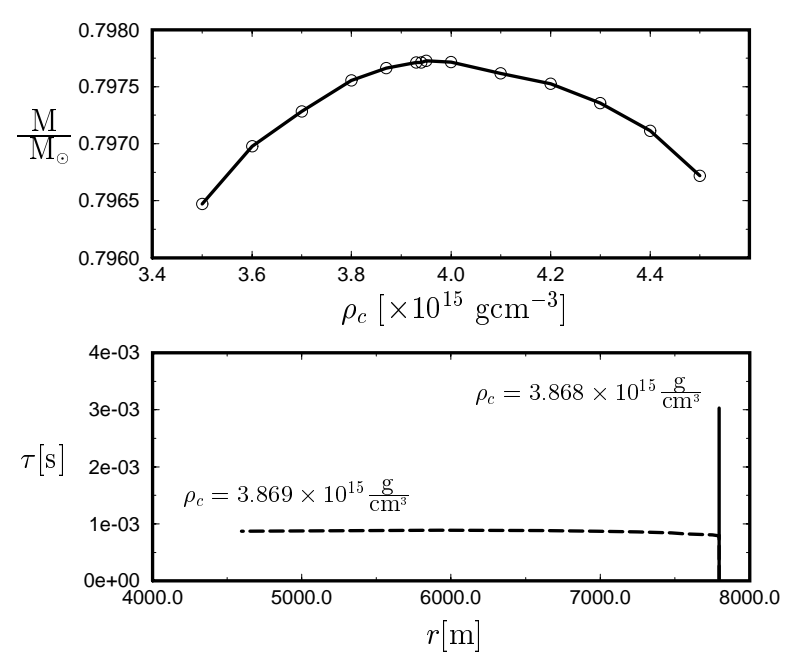

Fig. 3. Test case for determining the onset of instability in a neutron star. The upper panel shows the mass against the central density $\rho_{\mathrm{c}}$. Models with $\rho_{\mathrm{c}}>3.96 \times 10^{15} \mathrm{~g} \mathrm{~cm}^{-3}$ should be dynamically unstable. In the lower panel the surface radii is plotted as a function of time for two models with only slightly different central density. While the model with $\rho_{\mathrm{c}}=3.868 \times$ $10^{15} \mathrm{~g} \mathrm{~cm}^{-3}$ is stable and its radius is kept constant in time ( solid line), the one with $\rho_{\mathrm{c}}=3.869 \times 10^{15} \mathrm{~g} \mathrm{~cm}^{-3}$ collapses to a black hole in less than $1 \mathrm{~ms}$.

Fig. 2. Spacetime diagram of the collapse of a homogeneous dust sphere of radius unity (in arbitrary units) and of an additional dust shell, initially at rest and located at $r=1.4$. The vertical axis shows the advance of local proper time $\tau(r)$, i.e., the time measured by observers at fixed radius $r$. The trajectories of three outgoing lightrays (emitted at $\tau=$ $1.3978,1.4278,1.4578$ ) are shown as they escape from the forming black hole. Due to the gravitational distortion of the spacetime, lightrays are severely delayed. In such a situation most evolution codes are likely to experience problems when the event horizon is about to form due to finite numerical precision. Without taking additional measures it is not possible to compute the dotted section of the accreting mass shell. The dashed sections of the spacetime diagram are already inside the event horizon and are causally disconnected from distant observers.

\subsection{Tests of the numerical code}

For testing the accuracy of the code we use spherical neutron star configurations as there exist either previous results or analytic estimates that we can use. Convergence tests show that the code is second order accurate in space and time except at the center of the star. As the center is a local extremum, the numerical schemes we use (which belong to the so-called total variation diminishing class) are only first order accurate there. The code can keep a polytropic model of a neutron star in equilibrium for very long-term evolutions (roughly 200 light-crossing times).

Determining the onset of instability of stellar models by probing different central densities provides a very strong test for numerical codes. In order to do this, a sequence of neutron star equilibrium configurations is constructed by solving the TOV equations for several central densities. Only those configurations where $\frac{\mathrm{d} M}{\mathrm{~d} \rho_{\mathrm{c}}}>0$ are dynamically stable. In order to benchmark our results we use the same model as Baumgarte et al. (1995), namely a $5 / 3$ polytrope with $K=5.38 \times 10^{9}$ (in cgs units) with a mass of $0.79 M_{\odot}$. In Fig. 3 the dependence of $M$ on $\rho_{\mathrm{c}}$ is shown (upper panel). The maximum mass is obtained at $\rho_{\mathrm{c}}=3.96 \times 10^{15} \mathrm{~g} \mathrm{~cm}^{-3}$. The lower panel in Fig. 3 shows the surface radii of two different models having almost the same central density. The model with $\rho_{\mathrm{c}}=3.868 \times 10^{15} \mathrm{~g} \mathrm{~cm}^{-3}$ remains stable (solid line), while the model with $\rho_{\mathrm{c}}=3.869 \times 10^{15} \mathrm{~g} \mathrm{~cm}^{-3}$ collapses (dashed line). This is exactly the same numerical value quoted by Baumgarte et al. (1995). Although the determination of the critical density is only accurate at $2.3 \%$, the corresponding mass is identical up to the first three significant figures.

The accuracy of the code has also been measured by computing the frequencies of the radial modes of pulsations of a spherical neutron star. The numerical evolution of an initially static star is influenced by the local truncation error of the hydrodynamic scheme. These errors excite low amplitude radial pulsations whose frequencies can be measured by Fourier transforming the time evolution data of any fluid variable. Since in our code these frequencies are measured in retarded time coordinates (and not in terms of local proper time) one has to correct them by a redshift factor. The results can be compared to computations of linear normal modes (as an eigenvalue problem) or with results of other nonlinear codes. Our results agree very well with the frequencies computed by Font et al. (2000). Using 200 equidistant radial zones, the fundamental 

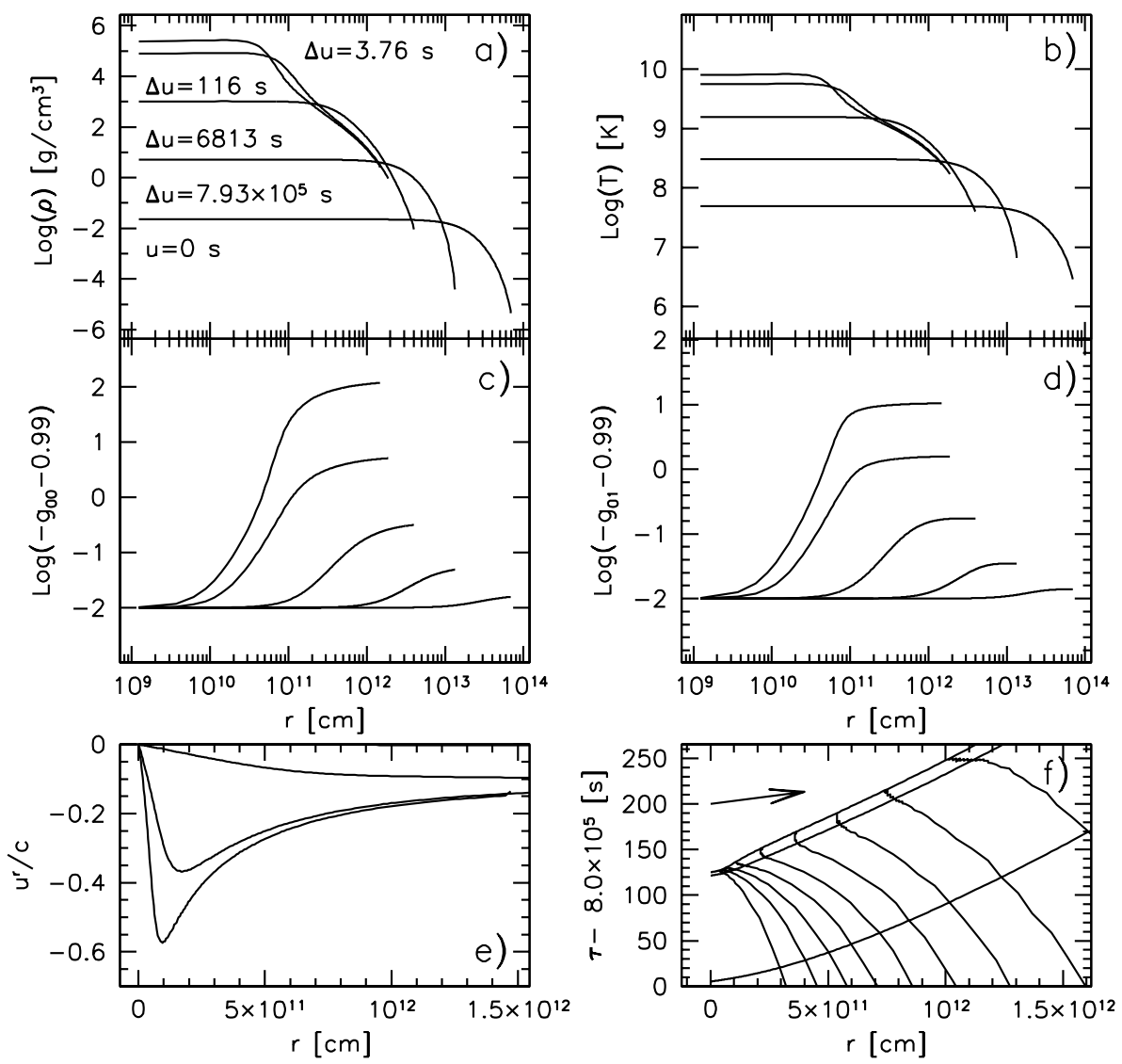

Fig. 4. Snapshots of $\rho, T, g_{00}, g_{01}$ and $u^{r}$ are shown for a $5 \times 10^{5} M_{\odot}$ SMS. The coordinate time interval $\Delta u$ elapsed between adjacent profiles diminishes since the evolution accelerates. The coordinate time is exactly equal to the proper time $\tau$ elapsed at the center of the star. The deviation of the metric from Minkowski spacetime, $g_{0 i}=-1$, is shown in the middle panels. As it is not possible to display a zero deviation in a logarithmic plot, the deviation from $g_{00}=-0.99$ is shown. In these plots $\log \left(-g_{0 \mu}-0.99\right) \equiv-2$ for $\mu \in\{0,1\}$ represents flat spacetime. The plot on the lower right side shows the location of mass shells $\left(\Delta M=5 \times 10^{4} M_{\odot}\right)$ versus local proper time $\tau$. The lines intersecting with the mass shells are hypersurfaces of constant coordinate time $u$. They represent trajectories of outgoing lightrays. The arrow gives the slope of a lightray at $r \rightarrow \infty$. Gravity already causes a severe delay of outgoing lightrays. Notice the kink in the upper hypersurface very close to the center of the star indicating the formation of an apparent horizon.

radial mode and the first two harmonics are computed with an accuracy of $0.12 \%, 3 \%$ and $0.1 \%$, respectively.

\subsection{Evolution of collapsing SMS}

We now describe the dynamics of the collapse of a spherical supermassive star. Although we have computed the collapse of all models listed in Table 1, we focus our discussion on the $5 \times 10^{5} M_{\odot}$ star, which represents our canonical model. Since all SMS are very closely approximated by 4/3-polytropes, the evolution of stars of different masses involves different length and time scales, but it is otherwise very similar.

Figure 4 shows the evolution of the density, temperature, metric components and velocity for the $5 \times 10^{5} M_{\odot}$ SMS. The collapse lasts $8 \times 10^{5} \mathrm{~s}(\approx 9.3$ days $)$ and the central density increases by a factor of $1.08 \times 10^{7}$. The initial configuration can be well described by Newtonian theory, as $g_{00}(R)=-1.0058$ at the surface of the star $R$, which deviates from the value in Minkowski spacetime, $g_{00}=-1$, by only $0.58 \%$. At the end of the simulation the final configuration has become highly relativistic and $g_{00}(R)=-119$. According to the relation $\mathrm{d} \tau^{2}=\left(e^{2 \beta} V / r\right) \mathrm{d} u^{2}$, this corresponds to a time dilation factor of about 11. In order to visualize this dramatic increase, Fig. 4 displays the deviation from flat spacetime logarithmically. In all simulations the center of the star is chosen to resemble Minkowski geometry, e.g. the boundary condition for $\beta$ is chosen as $\beta(r \equiv 0)=0$. All hydrodynamic quantities virtually freeze at the end of the simulation while the metric quantities start to grow very rapidly when black hole formation is about to begin. The maximum radial velocity encountered is about $-0.57 c$ at the very end of the simulation. The velocity profile is proportional to $r$ up to $r \approx 5.6 \times 10^{10} \mathrm{~cm}$, which corresponds to about $25 \%$ of the star's mass.

The panel on the lower right side of Fig. 4 shows the local proper time $\tau(r)$ vs. the position of mass shells enclosing fixed fractions of the total mass of the star. Between two lines the enclosed mass increases by $10 \%$ of the total 
mass of the star. The three lines intersecting the trajectories of the mass shells represent hypersurfaces of constant coordinate time $u$. They can be interpreted as the trajectories of outgoing lightrays. The arrow indicates the slope of a lightray at asymptotically large radii, i.e., in Minkowski spacetime. Thus, lightrays are severely delayed due to the distortion of the spacetime. By comparing the position of the mass shell enclosing $90 \%$ of the mass of the star with the Schwarzschild radius of a $5 \times 10^{5} M_{\odot}$ star, $R_{\mathrm{s}}=1.47 \times 10^{11} \mathrm{~cm}$, it becomes clear that the star still has to contract to less than one sixth of its current radius.

The density profiles in Fig. 4 are self-similar, i.e., the collapse proceeds homologously. A perfect homologous collapse is found until the central density has increased by a factor 798 corresponding to a central density $\rho_{\mathrm{c}} \approx 18 \mathrm{~g} \mathrm{~cm}^{-3}$. At $\rho_{\mathrm{c}} \approx 10^{3} \mathrm{~g} \mathrm{~cm}^{-3}$ the deviation from homology is evident. Goldreich \& Weber (1980) found analytically that in Newtonian theory a star with polytropic index $\Gamma=4 / 3$ should contract strictly homologously. The deviation from homologous collapse observed in the simulation is mainly due to effects of general relativity: time dilation results in a slower advance of local proper time $\tau(r)$ near the center of the star, and the proper volume element $\sqrt{-g} \mathrm{~d} r$ is larger than in Newtonian gravity. Therefore the density profiles in Fig. 4 tend to become steeper. Another contribution comes from the use of detailed microphysics. Due to copious $\mathrm{e}^{-} \mathrm{e}^{+}$pair production at the center of the star, where temperatures are highest, the increase of the internal energy is used to create the rest mass of the leptons instead of generating additional pressure. The EOS is thus softened, i.e. the adiabatic index is reduced, and the star tends to become more centrally condensed. However, since the deviation from homology is already apparent when the central temperatures are lower than $10^{9} \mathrm{~K}$ (see Fig. 4), $\mathrm{e}^{-} \mathrm{e}^{+}$pair creation can be considered as a minor contribution.

The evolution of SMS of different masses is found to be qualitatively very similar. The mass essentially affects the evolution only as a scaling parameter, except for the effects of neutrino emission. In all models of our sample, a black hole forms from the innermost $25 \%$ of the total stellar mass, and thus, the location of the innermost apparent horizon is proportional to $M$. In addition, the velocity profiles at the onset of black hole formation are found to be nearly identical. The timescale $\Delta \tau$ of neutrino emission, $\Delta \tau \approx R_{\mathrm{S}} / u^{r}\left(R_{\mathrm{S}}\right)$, is thus proportional to the stellar mass $M$ (see also Fig. 7). The dependence of the final core temperature on the mass is found to be $T_{\mathrm{c}} \sim M^{-0.5}$ (Shi \& Fuller 1998).

\subsection{Neutrino emission}

As already mentioned, three different kinds of thermal processes of neutrino production are included in the simulations: $\mathrm{e}^{+} \mathrm{e}^{-}$-pair annihilation, photo-neutrino emission, and plasmon decay. The neutrino generation rates in thermodynamic equilibrium depend only on the temperature

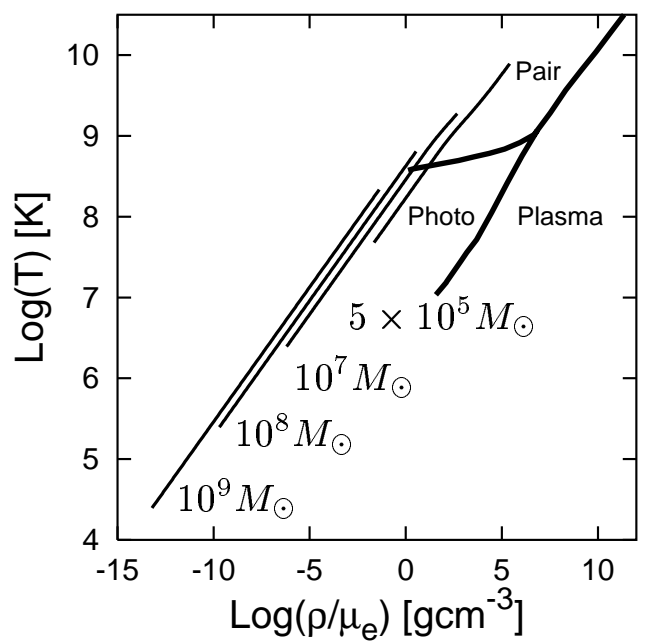

Fig. 5. Evolutionary tracks of several simulated SMS in the $\rho-T$-plane. More massive stars develop lower final core temperatures at the onset of black hole formation.

and density of the matter. Figure 5 shows the evolutionary tracks, including effects due to electron-positron pair creation in the stellar gas at $T>10^{9} \mathrm{~K}$, for our sample of stars in the range of $5 \times 10^{5} M_{\odot}-10^{9} M_{\odot}$.

From Fig. 5 it is obvious that the plasmon decay process is negligible for the simulations. For stars with masses below $5 \times 10^{8} M_{\odot}$ the pair creation process will be the most important process, as most of the energy is released in the last decade of central density increase. Black hole formation prevents stars with masses larger than $10^{9} M_{\odot}$ from entering the region dominated by pair annihilation. Neutrinos are then emitted primarily by the photo-neutrino process. Since the neutrino emission rate per volume for pair annihilation is $Q_{\nu} \sim T^{9}\left[\mathrm{erg} / \mathrm{s} / \mathrm{cm}^{3}\right]$ (Itoh et al. 1996), one can already infer from Fig. 5 that the total energy release in form of neutrinos decreases with increasing stellar mass.

As the volume increases $\left(\sim r^{2} \mathrm{~d} r\right)$ and the temperature decreases with radius, most neutrinos are emitted from a narrow spherical shell deep inside the star. Figure 6 depicts the radial dependence of the differential neutrino luminosities, $\mathrm{d} L_{\nu \bar{\nu}}(r) / \mathrm{d} r=4 \pi r^{2} Q_{\nu}$, for four models of our sample at various epochs (represented by the different lines for each star). The shells are centered at a radius $R_{\nu}$, at which the differential luminosity has a maximum. The total (unredshifted) neutrino luminosity for all flavors

$L_{\nu \bar{\nu}}=L_{\nu_{\mathrm{e}}}+L_{\bar{\nu}_{\mathrm{e}}}+L_{\nu_{\mu}}+L_{\bar{\nu}_{\mu}}+L_{\nu_{\tau}}+L_{\bar{\nu}_{\tau}}$,

is given by

$L_{\nu \bar{\nu}}(t)=\int_{0}^{\infty} 4 \pi r^{2} Q_{\nu}(T(r, t)) \mathrm{d} r$.

Figure 7 shows the computed neutrino luminosities $L_{\nu \bar{\nu}}\left(\tau_{\infty}\right)$ as seen by an observer at infinity for the collapse of different SMS. For each stellar mass the bare and the redshifted neutrino luminosities are depicted. It is possible to identify the moment in time when gravitational 


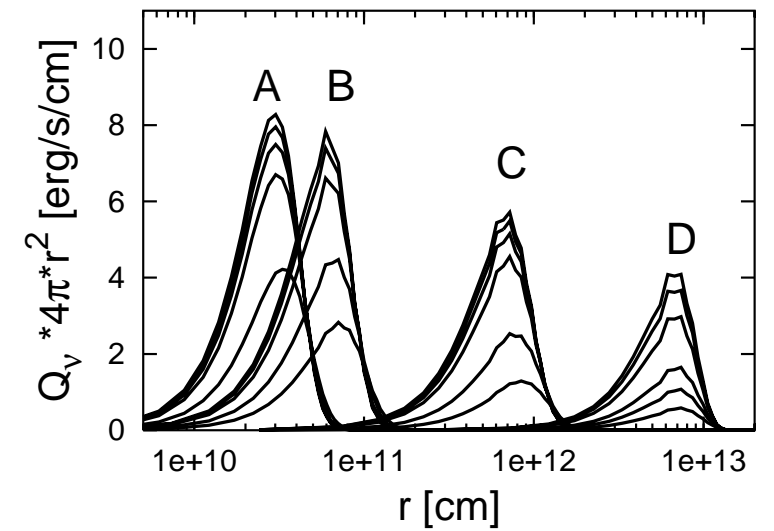

Fig. 6. Radial dependence of the (scaled) differential neutrino luminosities $\mathrm{d} L_{\nu \bar{\nu}}(r) / \mathrm{d} r$ for four SMS: (A) $5 \times 10^{5} M_{\odot}$, (B) $10^{6} M_{\odot},(\mathrm{C}) 10^{7} M_{\odot}$ and (D) $10^{8} M_{\odot}$. The various lines indicate different epochs for each model (increasing upwards). The upper curves correspond to the end of the simulations. The scale factors used are $5 \times 10^{44}$ (A), $10^{44}$ (B), $2 \times 10^{41}(\mathrm{C})$ and $2 \times 10^{36}(\mathrm{D})$, respectively. The main fraction of the neutrinos is emitted around a radius $R_{\nu}$, which is much smaller than the radius of the star at the end of the simulation $\left(\sim 1.47 \times 10^{12} \mathrm{~cm}\right.$ for the $\left.5 \times 10^{5} M_{\odot} \mathrm{SMS}\right)$.

redshift overcompensates the increasing neutrino energy release rates caused by growing temperatures. At the end of the simulation the luminosities are redshifted by more than two orders of magnitude.

The total energy release in form of neutrinos during the collapse is shown in Fig. 8 as a function of the stellar mass. The data points can be approximated by two different power laws. Doppler shift and gravitational redshift reduce the plotted values by approximately a factor of five. We find that for a $5 \times 10^{5} M_{\odot}$ SMS the total radiated energy amounts to $3.0 \times 10^{56}$ ergs, corresponding to an efficiency for converting rest mass energy to neutrino energy of $3.4 \times$ $10^{-4}$. This efficiency is depicted in Fig. 9 and defined as the (unredshifted) energy released in neutrinos divided by the total rest-mass energy of the star. After the formation of the black hole and after we stopped our simulations, we do not expect a significant fraction of the neutrino energy to be emitted. The reasons for this expectation will be discussed in some detail below. Therefore the efficiency is normalized to the rest-mass energy of the whole star, $M c^{2}$.

These results are in good agreement with the simulation of Woosley et al. (1986), who included neutrino transport during the collapse of a $5 \times 10^{5} M_{\odot}$ SMS with zero metallicity. They found a total energy output of $2.6 \times 10^{56}$ ergs in form of electron antineutrinos. As the neutrino energy release in the pair-dominated region depends on the ninth power of the temperature, most neutrinos are emitted, in the case of a $5 \times 10^{5} M_{\odot} \mathrm{SMS}$, from the very center of the star within the last $\Delta \tau_{\infty} \approx 10 \mathrm{~s}$ of the collapse before a black hole forms. Even slightly different core temperatures at the onset of black hole formation can result in a large difference of the total energy release.

The broken power law behavior can be understood in terms of simple considerations. Assuming adiabatic

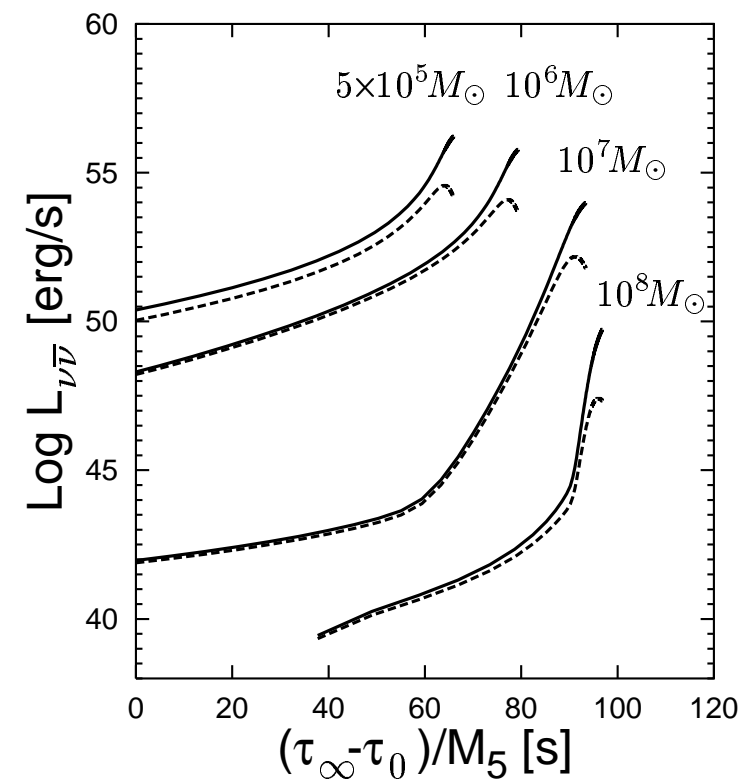

Fig. 7. Time evolution of the neutrino luminosities, bare (solid) and redshifted (dashed), for several stars in the range $5 \times 10^{5} M_{\odot}-10^{8} M_{\odot}$. Quantity $\tau_{\infty}$ represents the proper time for an observer at infinity. This time is measured relative to the overall collapse timescale $\tau_{0}=\left\{8.05 \times 10^{5} \mathrm{~s}, 1.7 \times 10^{6} \mathrm{~s}\right.$, $\left.8.05 \times 10^{7} \mathrm{~s}, 3.2 \times 10^{9} \mathrm{~s}\right\}$ and scaled by $M_{5}=M /\left(10^{5} M_{\odot}\right)$. As expected, the maximum luminosity decreases with the mass of the star. The change of the slope of the neutrino light curves at luminosities $\sim 10^{43} \mathrm{erg} \mathrm{s}^{-1}$, visible in the models with $10^{7} M_{\odot}$ and $10^{8} M_{\odot}$, is due to the transition from photo-neutrino dominated to pair-dominated emission. The rates of energy release by neutrino production is much more sensitive to the temperature in the latter case.

contraction, $T_{\nu} \sim M^{-0.5}$, a typical timescale of neutrino emission $\Delta \tau \sim M$ (see Fig. 7), a radiating volume with radius $R_{\nu} \sim R_{\mathrm{s}} \sim M$ (see Fig. 6) and a neutrino energy emissivity proportional to the ninth power of the temperature (pair annihilation) at $R_{\nu}$ (Itoh et al. 1996), the dependence of the total energy release on the mass of the star is given by $E_{\nu} \sim M^{-0.5}$ (Fuller \& Shi 1998). This dependence describes very well the results of the simulations in the low mass range (high core temperatures $\left.T_{\nu}>3 \times 10^{9} \mathrm{~K}\right)$. Stars with masses above $5 \times 10^{7} M_{\odot}$ reach core temperatures just below $10^{9} \mathrm{~K}$ where the neutrino emissivity is proportional to the 20th power of the temperature because of the onset of $\mathrm{e}^{+} \mathrm{e}^{-}$-pair creation (Itoh et al. 1996). The total energy release is thus proportional to $E_{\nu} \sim M^{-6}$, which is again in good agreement with the simulations. In summary, one finds for the total neutrino luminosities the limiting relations

$L_{\nu \bar{\nu}} \sim Q_{\nu} \frac{4 \pi}{3} R_{\nu}^{3} \sim\left\{\begin{array}{cl}M^{-1.5} & \left(10^{5} \lesssim \frac{M}{M_{\odot}} \lesssim 5 \times 10^{6}\right), \\ M^{-7} & \left(5 \times 10^{7} \lesssim \frac{M}{M_{\odot}} \lesssim 10^{8}\right),\end{array}\right.$

and for the total energy release (not redshifted)

$E_{\nu} \sim\left\{\begin{array}{cc}M^{-0.5} & \left(10^{5} \lesssim \frac{M}{M_{\odot}} \lesssim 5 \times 10^{6}\right), \\ M^{-6} & \left(5 \times 10^{7} \lesssim \frac{M}{M_{\odot}} \lesssim 10^{8}\right) .\end{array}\right.$ 


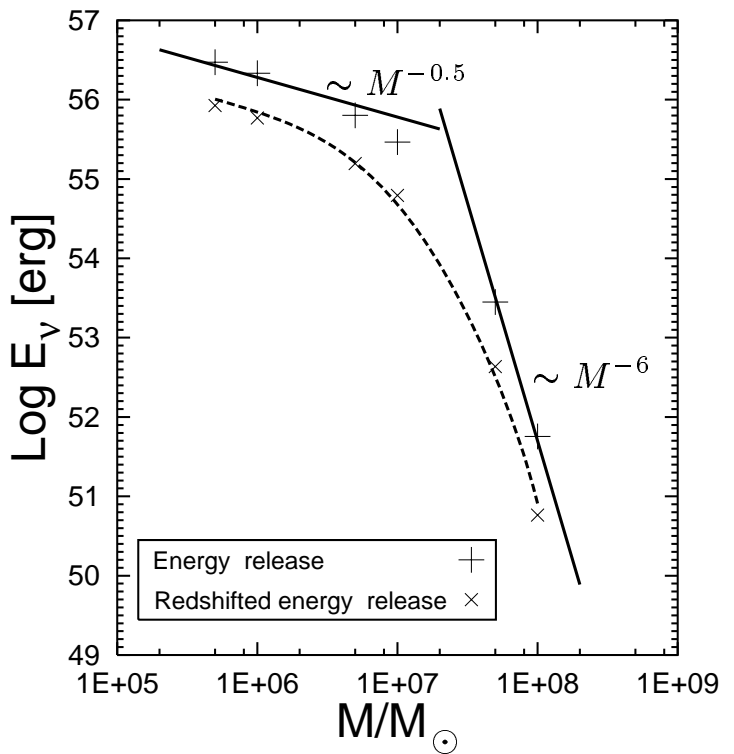

Fig. 8. The total energy release in form of neutrinos against the mass of the SMS. The symbols indicate the computed models. The data points can be described by a broken power law.

\section{4. $\nu \bar{\nu}$-annihilation and energy deposition}

In 1998 Fuller \& Shi proposed the collapse of a SMS as a possible mechanism to trigger GRBs. In order to check this idea quantitatively, we calculate in this section the amount of energy that is deposited by $\nu \bar{\nu}$-annihilation in our collapse models of SMS. In the following, the results obtained for the neutrino emission in the previous section will also be used to estimate the spatial distribution of the deposited energy.

Our hydrodynamic simulations indicate that, provided nuclear burning is unimportant, the gravitational collapse of a SMS proceeds rather smoothly, involving no shocks. The collapse leads to the formation of a black hole consisting of the innermost $25 \%$ of the star's mass, onto which the rest of the stellar matter accretes. At the same time an energy up to $\mathcal{O}\left(10^{57}\right)$ ergs is released in form of neutrinos. A small fraction of this energy is deposited within the star. In order to accelerate stellar matter to ultrarelativistic speeds, the energy deposition must take place in an almost baryon-free region, i.e. very close to the surface of the star, or in a baryon-poor funnel along the polar axis in case of a rotating SMS. The kinetic energy of the ultrarelativistic flow could then be converted to $\gamma$-rays by cyclotron radiation and/or by the inverse Compton process.

Such ultrarelativistic flows from SMS might be powered by the energy deposition due to $\nu \bar{\nu}$-annihilation (Fuller \& Shi 1998),

$\nu_{\mathrm{e},(\mu, \tau)}+\bar{\nu}_{\mathrm{e},(\mu, \tau)} \rightarrow \mathrm{e}^{-}+\mathrm{e}^{+}$,

since this process is capable to deposit a large amount of energy even in vacuum. The duration of the strong neutrino emission from the collapse of a $5 \times 10^{5} M_{\odot}$ SMS is about $30 \mathrm{~s}$ for an observer at infinity (see Fig. 7) and thus

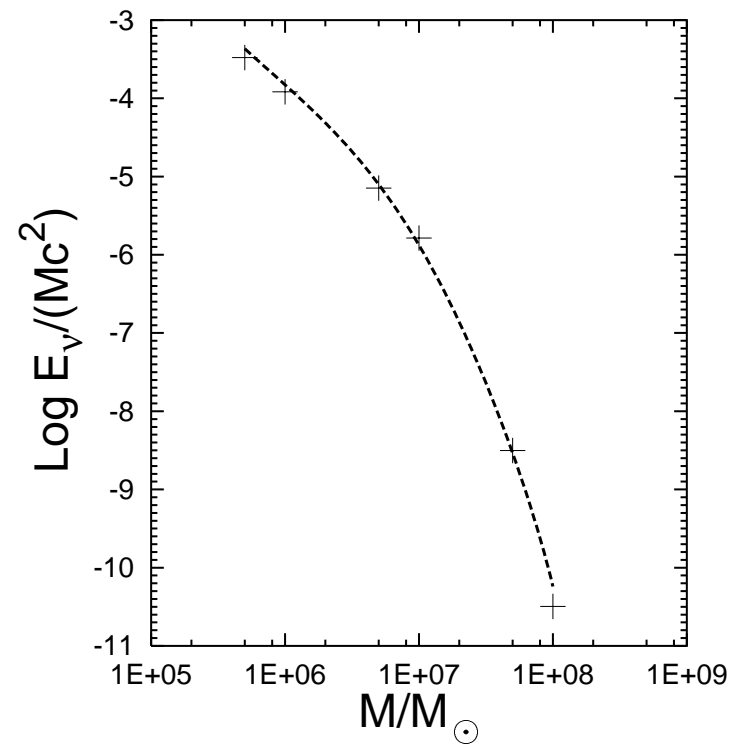

Fig. 9. Efficiency for converting the rest mass of a SMS to neutrinos. As less massive stars evolve to higher core temperatures, both the total energy emitted in form of neutrinos and the conversion efficiency rise with decreasing mass.

in agreement with the duration of observed long GRBs (Piran 1999).

For a neutrino-radiating sphere of radius $R_{\nu}$, the volume-integrated energy deposition rate due to $\nu \bar{\nu}$ annihilation above $R_{\nu}$ is (Goodman et al. 1987; Cooperstein et al. 1987)

$\dot{E}_{\mathrm{dep}}^{\mathrm{e},(\mu, \tau)}=\frac{A}{R_{\nu}} L_{\nu_{\mathrm{e}(\mu, \tau)}} L_{\bar{\nu}_{e(\mu, \tau)}}\left(\frac{\left\langle\epsilon_{\bar{\nu}}^{2}\right\rangle}{\left\langle\epsilon_{\bar{\nu}}\right\rangle}+\frac{\left\langle\epsilon_{\nu}^{2}\right\rangle}{\left\langle\epsilon_{\nu}\right\rangle}\right)$,

with $A=K_{\mathrm{e},(\mu, \tau)} G_{\mathrm{F}}^{2} / 9 \pi c$. Here $G_{\mathrm{F}}^{2}=2.066 \times$ $10^{-32} \mathrm{~cm}^{2} / \mathrm{erg}^{2}$ is the Fermi constant, $L_{\nu_{\mathrm{e}(\mu, \tau)}}$ and $L_{\bar{\nu}_{\mathrm{e}(\mu, \tau)}}$ are the neutrino and antineutrino luminosities at $R_{\nu},\left\langle\epsilon_{\nu}\right\rangle$ and $\left\langle\epsilon_{\nu}^{2}\right\rangle$ are the energy moments of the neutrino phase space distribution, evaluated by using a fitting formula for the pair neutrino spectrum as given by Shi \& Fuller (1998). $K_{\mathrm{e},(\mu, \tau)}$ is a dimensionless constant, which, according to the standard model of weak interactions, is given by

$K_{\mathrm{e},(\mu, \tau)}=\left\{\begin{array}{l}\frac{1}{6 \pi}\left(1+4 \sin ^{2} \theta_{\mathrm{W}}+8 \sin ^{4} \theta_{\mathrm{W}}\right) \text { for } \nu_{\mathrm{e}} \bar{\nu}_{\mathrm{e}}, \\ \frac{1}{6 \pi}\left(1-4 \sin ^{2} \theta_{\mathrm{W}}+8 \sin ^{4} \theta_{\mathrm{W}}\right) \text { for } \nu_{\mu, \tau} \bar{\nu}_{\mu, \tau},\end{array}\right.$

with $\theta_{\mathrm{W}}$ being the Weinberg angle $\left(\sin \theta_{\mathrm{W}}=0.23\right)$. Assuming that most neutrinos are produced by $\mathrm{e}^{-} \mathrm{e}^{+}$-pair annihilation it is possible to compute the total energy deposition rate $\dot{E}_{\mathrm{dep}}^{\mathrm{e}(\mu, \tau)}$ as

$\dot{E}_{\mathrm{dep}}^{\mathrm{e},(\mu, \tau)}=2 A\left(\frac{K_{\mathrm{e},(\mu, \tau)}}{K_{\mathrm{e}}+2 K_{(\mu, \tau)}} \frac{L_{\nu \bar{\nu}}}{2}\right)^{2} \frac{41.225}{5.769} \frac{T_{\nu}}{R_{\nu}}$,

where $T_{\nu}$ is the temperature at $R_{\nu}$ and $L_{\nu \bar{\nu}}$ is the total neutrino luminosity.

For the sake of simplicity and comparison with Fuller \& Shi (1998), all effects due to relativistic redshift or general 


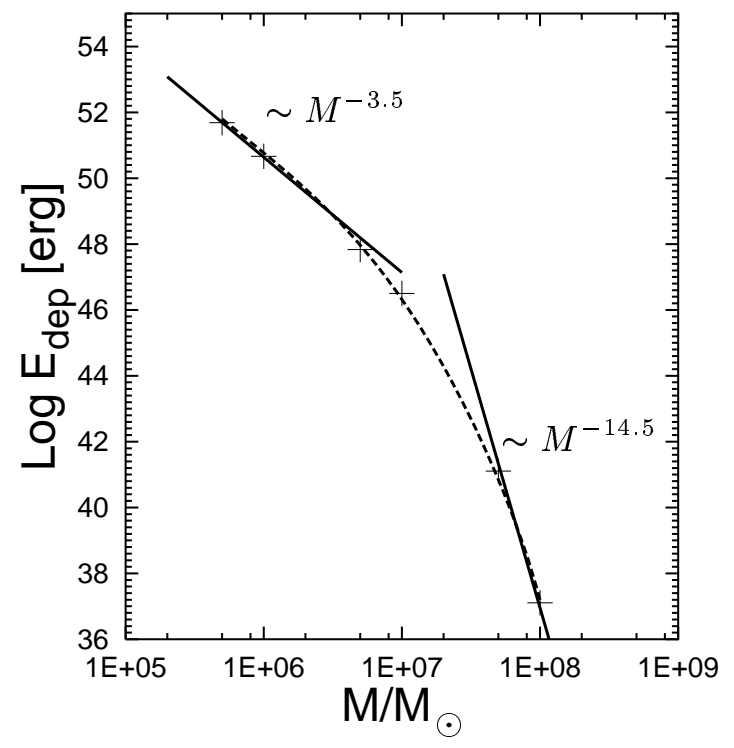

Fig. 10. The total neutrino energy deposition as a function of the mass of the SMS. Due to decreasing neutrino luminosities the total energy deposited within the star decreases rapidly with the mass of the star. The dependences on both extremes of the considered mass range can be understood by simple considerations (see text for details).

relativistic ray bending were neglected in the evaluation of the $\nu \bar{\nu}$-annihilation. Towards the end of our simulations, however, they play an increasingly important role. For the $5 \times 10^{5} M_{\odot}$ star, the main neutrino emitting region is located near $3 \times 10^{10} \mathrm{~cm}$. According to Fig. $4 \mathrm{c}$, the gradient of the redshift factor is steepest around that radius, close to which the apparent horizon forms. This gradient will become even steeper and grow even faster as the collapse proceeds. Gravitational redshift will therefore quench the neutrino luminosity, also in the layer where most of the neutrinos annihilate. At larger radii the temperature is too low to allow for strong neutrino production. This will in particular be true during the phase when the forming black hole swallows the remaining $\sim 75 \%$ of the SMS, because there is no resistance to the infalling gas, and compressional heating will therefore stay moderate. For this reason it is very unlikely that the accretion of the rest of the star's mass onto the seed black hole will yield a large contribution to the total neutrino emission and energy release by $\nu \bar{\nu}$-annihilation.

The resulting energy deposition for different SMS is plotted in Fig. 10. The corresponding energy deposition efficiency $E_{\mathrm{dep}} / E_{\nu}$ is shown in Fig. 11. The dependence of the deposited energy on the mass of the star at both ends of the considered mass range is $E_{\text {dep }} \sim L_{\nu \bar{\nu}}^{2} T_{\nu} \Delta \tau / R_{\nu} \sim$ $L_{\nu \bar{\nu}}^{2} M^{-0.5}$, where $\Delta \tau$ is the timescale of the emission. Therefore, using Eq. (16)

$E_{\mathrm{dep}} \sim\left\{\begin{array}{cc}M^{-3.5} & \left(10^{5} \lesssim \frac{M}{M_{\odot}} \lesssim 5 \times 10^{6}\right), \\ M^{-14.5} & \left(5 \times 10^{7} \lesssim \frac{M}{M_{\odot}} \lesssim 10^{8}\right) .\end{array}\right.$

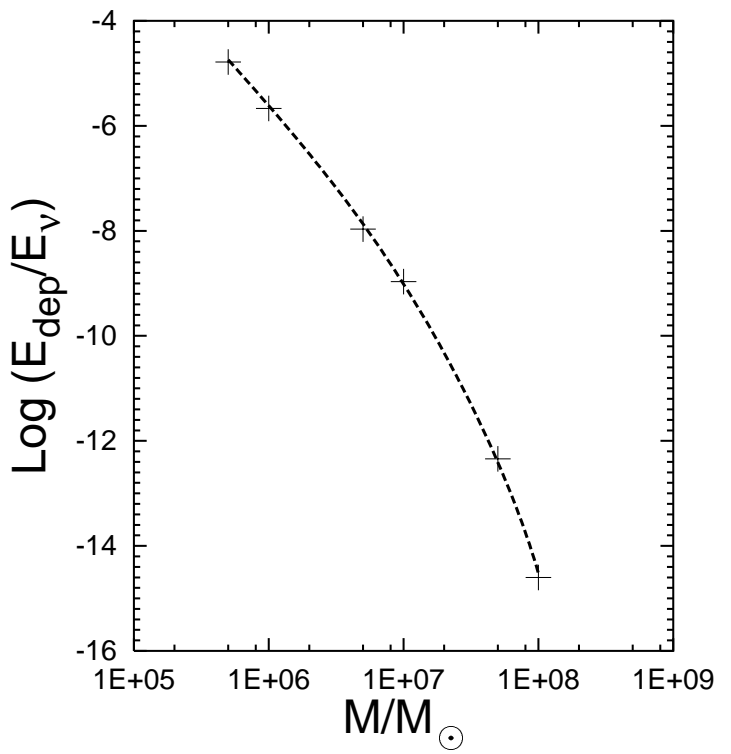

Fig. 11. Efficiency for conversion of neutrino energy to pair plasma by $\nu \bar{\nu}$-annihilation.

Fuller \& Shi (1998) gave analytic estimates for the expected neutrino emission from the collapse of a SMS. They employed a simple model to deduce the final average core temperature $T_{\mathrm{c}}$ as a function of the mass of the homologously collapsing core $M^{\mathrm{HC}}$. Assuming neutrino energy release rates $Q_{\nu} \sim T_{\mathrm{c}}^{9}$ and a radiating sphere with a radius equal to the Schwarzschild radius, $R_{\mathrm{S}}=2 G M^{\mathrm{HC}} / c^{2}$, for the homologously collapsing mass, their estimate for the characteristic neutrino luminosity is

$L_{\nu \bar{\nu}} \approx 5 \times 10^{57}\left(M_{5}^{\mathrm{HC}}\right)^{-3 / 2} \mathrm{erg} / \mathrm{s}$,

where $M_{5}^{\mathrm{HC}}=M^{\mathrm{HC}} / 10^{5} M_{\odot}$. Furthermore, assuming a typical emission timescale of $\Delta \tau \approx M_{5}^{\mathrm{HC}}$ s, their result for the energy deposited above a radius $r$ is

$E_{\text {dep }} \approx 2.5 \times 10^{54}\left(M_{5}^{\mathrm{HC}}\right)^{-3.5}\left(R_{\mathrm{s}} / r\right)^{5}$ erg.

The simulation presented in the previous section shows that in a $5 \times 10^{5} M_{\odot}$ SMS an inner core with $25 \%$ of the stellar mass is collapsing homologously, i.e. $M_{5}^{\mathrm{HC}}=1.25$. Table 2 shows the estimates of Fuller \& Shi in comparison to the results of our simulation. Since the physics involved in the description of SMS is well known, all their basic assumptions have been confirmed by our simulation: (1) The core temperature $T_{\mathrm{c}} \sim T_{\nu} \sim M^{-0.5}$, (2) the radius of the radiating sphere $R_{\nu} \sim M$, (3) the time scale of the emission $\Delta \tau \sim M$, and (4) the computed neutrino energy generation rates do not differ by more than $25 \%$ if they are evaluated for the same temperature $T \approx 10^{10} \mathrm{~K}$. The large discrepancy of the total deposited energy $E_{\text {dep }}$ is caused by the strong dependence of the energy deposition rate $\dot{E}_{\text {dep }}$ on $T_{\nu}$ and $R_{\nu}, \dot{E}_{\text {dep }} \sim T_{\nu}^{19} R_{\nu}^{5}$ (see Eq. (20) with Eq. (16)). Misjudging slightly both the radius $R_{\nu}$ of the radiating sphere and the temperature $T_{\nu}$ at that radius leads to a very large overestimation of the energy deposition rate. Furthermore, the way in which we compute the 
Table 2. Comparison of the analytic model of Fuller \& Shi (1998) with the results of our numerical simulation. Values are missing where the approximations by Fuller \& Shi are not applicable or do not provide information. Overestimating the temperature $T_{\nu}$ at the neutrino-radiating sphere $R_{\nu}$ leads to a large error in the rate of energy release by neutrino emission $Q_{\nu}$ and thus in the energy deposition $E_{\mathrm{dep}}$ at $3 R_{\nu} \leq r<\infty$.

\begin{tabular}{ccc}
\hline$M=5 \times 10^{5} M_{\odot}$ & Simulation & Fuller \& Shi \\
\hline$T_{\nu}[\mathrm{K}]$ & $8.0 \times 10^{9}$ & $11.6 \times 10^{9}$ \\
$R_{\nu}[\mathrm{cm}]$ & $3.0 \times 10^{10}$ & $3.8 \times 10^{10}$ \\
$Q_{\nu}\left(R_{\nu}\right)\left[\mathrm{erg} / \mathrm{s} / \mathrm{cm}^{3}\right]$ & $4.1 \times 10^{23}$ & $1.2 \times 10^{25}$ \\
$L_{\nu \bar{\nu}}[\mathrm{erg} / \mathrm{s}]$ & $1.7 \times 10^{56}$ & $3.6 \times 10^{57}$ \\
$E_{\mathrm{dep}}\left(r>R_{\nu}\right)[\mathrm{erg}]$ & $4.9 \times 10^{51}$ & - \\
$E_{\mathrm{dep}}\left(r>3 R_{\nu}\right)[\mathrm{erg}]$ & $8.0 \times 10^{48}$ & $4.7 \times 10^{51}$ \\
$M\left(r>3 R_{\nu}\right)$ & $\sim 0.65 M_{\mathrm{SMS}}$ & - \\
\hline
\end{tabular}

neutrino luminosities in our simulations, Eq. (15), is also more accurate than the formula employed by Fuller \& Shi, $L_{\nu \bar{\nu}}=Q_{\nu}\left(T_{\nu}\right) \frac{4 \pi}{3} R_{\nu}^{3}$. This, however, decreases the ratio of Fuller and Shi's neutrino luminosity to our luminosity by a factor of three, corresponding to a reduction of the disagreement of the energy deposition by $\nu \bar{\nu}$-annihilation by a factor of nine, to yield a remaining discrepancy of nearly a factor of 600 .

\section{Implication for gamma ray bursts}

The integral amount of deposited energy, $E_{\text {dep }} \approx 4.9 \times$ $10^{51} \mathrm{erg}$ in the case of a $5 \times 10^{5} M_{\odot}$ SMS, is at the lower end of the observed energy released in cosmic GRBs. All of this energy would have to be collimated into narrow jets with a collimation of $\delta \Omega / 4 \pi \lesssim 1 / 1000-1 / 100$ in order to explain an equivalent isotropic energy of $10^{53}$ to $\gtrsim 10^{54}$ erg as inferred for observed long-duration bursts with known cosmological redshifts (e.g., Frail et al. 2001). However, 99.837\% (Goodman et al. 1987) of this energy is deposited in a spherical layer deep inside the star at $R_{\nu} \leq r \leq 3 R_{\nu}$, and only a tiny fraction near the surface of the star, where excessive baryon loading could be avoided. Therefore ultrarelativistic ejection of matter with Lorentz factors $\gamma \gg 1$ cannot be expected in spherical models:

$\gamma \approx 1+\frac{E_{\mathrm{dep}}\left(r>R_{\nu}\right)}{M\left(r>R_{\nu}\right) c^{2}} \approx 1$.

We therefore conclude that energy deposition by $\nu \bar{\nu}$ annihilation in the spherical collapse of a $5 \times 10^{5} M_{\odot} \mathrm{SMS}$ does not meet the demands for being a successful central engine for a GRB.

A rotating SMS would have a reduced baryon density along the polar axis and the time scale of neutrino emission could be longer, because a disk stabilized by centrifugal forces would prolong the accretion of matter into a forming black hole. Thus, the conditions to fulfill the constraints of GRB models might appear more suitable in this respect. Nevertheless, as the energy release rates by neutrino emission and the energy deposition in nonrotating models are several orders of magnitude below the estimates of Fuller \& Shi, it still seems unlikely that the total energy release in a baryon-poor environment would be sufficiently large in rotating scenarios. However, an ultimate statement requires detailed numerical simulations of the gravitational collapse of rotating SMS.

As shown in Fig. 10 the total energy deposition depends strongly on the mass $E_{\text {dep }} \sim M^{-3.5}$. Therefore, more massive stars are less promising to meet the energy requirements of a GRB. Extrapolating the total deposited energy of a $5 \times 10^{5} M_{\odot}$ star to less massive stars, the expected value for a $10^{5} M_{\odot}$ SMS is $E_{\text {dep }} \approx$ $1.3 \times 10^{54} \mathrm{erg}$. The energy deposited within $\left[3 R_{\nu}, \infty\right]$ is a factor $1.63 \times 10^{-3}$ smaller and therefore of the order of $10^{51}$ ergs, which is at the lower end of the observed energy range. However the baryon loading problem is still critical. We have not attempted to simulate the evolution of such a star because in this case nuclear burning becomes relevant. The energy release in the form of neutrinos will also depend strongly on the fate of the star: If the energy release due to nuclear burning cannot inhibit black hole formation the neutrino emission increases with smaller stellar mass. If nuclear energy is liberated rapidly enough, the star will be destroyed in a thermonuclear explosion. Consequently, there will be much less neutrino emission, since the core temperature will be considerably lower. Only detailed numerical simulations of the collapse of such stars can determine the expected energy release and their final fate.

\section{Summary}

We have presented results of numerical simulations of the spherically symmetric gravitational collapse of supermassive stars. Such simulations were performed using a general relativistic hydrodynamics code. The coupled system of Einstein and fluid equations was solved adopting a spacetime foliation with outgoing null (characteristic) hypersurfaces. The code includes a tabulated equation of state which accounts for contributions from radiation, electrons, electron-positron pairs and baryonic gases. Energy losses by thermal neutrino emission were taken into account.

We were able to follow the collapse of SMS from the onset of instability up to the point of black hole formation. Several SMS with masses in the range of $5 \times 10^{5} M_{\odot}-10^{9} M_{\odot}$ were simulated, showing that an apparent horizon forms in all cases, enclosing the innermost $25 \%$ of the stellar mass. This is in good agreement with previous simulations by other authors. We did not attempt to follow the subsequent accretion of the remaining mass onto the central black hole because, due to finite numerical precision, the innermost apparent horizon is ultimately penetrated (see Figs. 2 and 4, in particular the little kink developing in the lightcones in frame (f) of the latter). This effect however, does not interfere with the main aim of our work, namely the computation of neutrino luminosities, because most neutrinos are emitted near the very center of the star. Therefore we did not try 
possible remedies to follow the evolution for longer times, such as excising the inner regions of the domain, in which the evolution essentially freezes, and imposing appropriate boundary conditions there.

The neutrino luminosities for several SMS models have been computed and it has been possible to understand the dependence of the total energy release in neutrinos, $E_{\nu}$, on the stellar mass $M$ in terms of simple scaling laws. Based on these results we have given estimates of the energy deposition by $\nu \bar{\nu}$-annihilation in the star. Our simulations show that for collapsing SMS with masses larger than $5 \times 10^{5} M_{\odot}$ this energy deposition is more than two orders of magnitude smaller than the estimates of Fuller \& Shi (1998). In addition, all of this energy is deposited deep inside the star and cannot drive relativistic outflows. Therefore, the spherical collapse of such a SMS does not meet the demands for being a successful GRB model.

Acknowledgements. All computations were performed on the NEC SX-5/3C supercomputer at the Rechenzentrum Garching. We want to thank M. Salaris who kindly provided us with his implementation of the energy production rates by neutrino processes as published by Itoh et al. (1996) and Haft et al. (1994). This work was supported in part by the SFB-375 "Astroparticle Physics" of the Deutsche Forschungsgemeinschaft.

\section{References}

Appenzeller, I., \& Fricke, K. 1972a, A\&A, 18, 10

Appenzeller, I., \& Fricke, K. 1972b, A\&A, 21, 285

Baumgarte, T. W., Shapiro, S. L., \& Teukolsky, S. A. 1995, ApJ, 443, 717

Baumgarte, T. W., \& Shapiro, S. L. 1999a, ApJ, 526, 937

Baumgarte, T. W., \& Shapiro, S. L. 1999b, ApJ, 526, 941

Bondi, H., van der Burg, M. J. G., \& Metzner, A. W. K. 1962 , Proc. R. Soc. London, Sect. A, 269, 21
Chandrasekhar, S. 1964, ApJ, 140, 417

Cooperstein, J., van den Horn, L. J., \& Baron, E. 1987, ApJ, 321, L129

Font, J. A., Stergioulas, N., \& Kokkotas, K. D. 2000, MNRAS, 313,678

Fowler, W. F. 1964, Rev. Mod. Phys., 36, 545

Frail, D. A., et al. 2001, preprint [astro-ph/0102282]

Fricke, K. 1973, ApJ, 183, 941

Fuller, G. M., \& Shi, X. 1998, ApJ, 502, L5

Fuller, G. M., Woosley, S. E., \& Weaver, T. A. 1986, ApJ, 307, 675

Genzel, R., Picho, C., Eckart, A., Gerhard, O. E., \& Ott, T. 2000, MNRAS, 317, 348

Goldreich, P., \& Weber, S. V. 1980, ApJ, 238, 991

Goodman, J., Dar, A., \& Nussinov, S. 1987, ApJ, 314, L7

Gómez, R., Laguna, P., Papadopoulos, P., \& Winicour, J. 1996, Phys. Rev. D, 54, 4719

Haft, M., Raffelt, G., \& Weiss, A. 1994, ApJ, 425, 222

Itoh, N., Hayashi, H., Nishikawa, A., \& Kohyama, Y. 1996, ApJS, 102, 411

Kippenhahn, R., \& Wigert, A. 1994, Stellar Structur and Evolution (Springer)

Kormendy, J. 2000, preprint [astro-ph/0007401]

Maoz, E. 1998, ApJ, 494, L181

Papadopoulos, P., \& Font, J. A. 2000a, Phys. Rev. D, 61, 024015

Papadopoulos, P., \& Font, J. A. 2000b, preprint [gr-qc/ 9912054]

Piran, T. 1999, Phys. Rep., 314, 575

Rees, M. J. 1984, Ann. Rev. Astr. Astrphys., 22, 471

Rees, M. J. 1998, in Black Holes and Relativistic Stars, ed. R. M. Wald (University of Chicago Press, Chicago)

Sachs, R. K. 1962, Proc. R. Soc. London, Sect. A, 270, 103

Shapiro, S. L., \& Teukolsky, S. A. 1979, ApJ, 234, L177

Shapiro, S. L., \& Teukolsky, S. A. 1985, ApJ, 298, 34

Shi, X., \& Fuller, G. M. 1998, ApJ, 503, 307

Thorne, K. S., \& Braginsky, V. B. 1976, ApJ, 204, L1 